\title{
Revisiting the influence of learning in predator functional response, how it can lead to shapes different from type III.
}

\author{
Octavio Bruzzone ${ }^{1}$, María Aguirre ${ }^{2}$, Jorge Hill ${ }^{3}$, Eduardo Virla ${ }^{4}$, and Guillermo Logarzo ${ }^{2}$ \\ ${ }^{1}$ INTA \\ ${ }^{2}$ Fundación para el Estudio de Especies Invasivas \\ ${ }^{3}$ Universidad Nacional de Tucumán Facultad de Agronomía y Zootecnia \\ ${ }^{4}$ PROIMI-Biotecnología, CONICET
}

September 25, 2021

\begin{abstract}
Abstract Predator/Parasitoid functional response is one of the main tools used to study predation behaviour, and in assessing the potential of biological control candidates. It is generally accepted that predator learning in prey searching and manipulation can produce the appearance of type III functional response. Holling proposed that in the presence of alternative prey, at some point the predator would shift the preferred prey, leading to the appearance of a sigmoid function that characterized that functional response. This is supported by the analogy between enzyme kinetics and functional response that Holling used as the basis for developing this theory. However, after several decades, sigmoidal functional responses appear in the absence of alternative prey in most of the biological taxa studied. Here, we propose modelling the effect of learning on the functional response by using the explicit incorporation of learning curves in the parameters of the Holling functional response, the attack rate (a), and the manipulation time (h). We then study how the variation in the parameters of the learning curves causes variations in the shape of the functional response curve. We found that the functional response product of learning can be either type I, II or III, depending on what parameters act on the organism, and how much it can learn throughout the length of the study. Therefore the presence of other types of curves should not be automatically associated with the absence of learning. These results are important from an ecological point of view because when type III functional response is associated with learning, it is generally accepted that it can operate as a stabilizing factor in population dynamics. Our results, to the contrary, suggest that depending on how it acts, it may even be destabilizing by generating the appearance of functional responses close to type I.
\end{abstract}

\section{Hosted file}

Bruzzone et al. 2021.pdf available at https://authorea.com/users/437302/articles/539036revisiting-the-influence-of-learning-in-predator-functional-response-how-it-can-lead-toshapes-different-from-type-iii 